\title{
Polaron formation and hopping in tantalate perovskite oxides: $\mathrm{NaTaO}_{3}$ and $\mathrm{KTaO}_{3}$
}

\author{
Hassan Ouhbi $\odot$ and Julia Wiktor ${ }^{*} *$ \\ Department of Physics, Chalmers University of Technology, SE-412 96 Gothenburg, Sweden
}

(Received 26 July 2021; revised 10 November 2021; accepted 10 December 2021; published 30 December 2021)

\begin{abstract}
Perovskite tantalates have become potential candidates for water splitting photocatalysts. Therefore, it is of importance to understand the behavior of the photoinduced excess charges in these materials. Herein, we investigate the formation of electron and hole polarons in $\mathrm{NaTaO}_{3}$ and $\mathrm{KTaO}_{3}$. We perform Perdew-Burke-Ernzerhof hybrid density functional $\operatorname{PBE} 0(\alpha)$ calculations, in which we define the fraction $\alpha$ of the Fock exchange by enforcing the Koopmans' condition, to properly account for self-interaction corrections in these calculations. We find that the hole polaron mainly localizes on one oxygen site in both materials, leading to a structural distortion where two Ta-O bonds are elongated. The electron polaron, on the other hand, localizes within one atomic plane and exhibits a two-dimensional electron gas nature. Finally, we find that the strong localization of holes leads to a low hole mobility at room temperature $\sim 2.94 \times 10^{-6} \mathrm{~cm}^{2} / \mathrm{Vs}$ and $\sim 1.87 \times 10^{-4} \mathrm{~cm}^{2} / \mathrm{Vs}$ for $\mathrm{KTaO}_{3}$ and $\mathrm{NaTaO}_{3}$, respectively.
\end{abstract}

DOI: 10.1103/PhysRevB.104.235158

\section{INTRODUCTION}

Green hydrogen production by photocatalytic water splitting is a promising technology. In order to make a photocatalyst-based system, the catalyst must have a band gap below $\sim 3 \mathrm{eV}$, to achieve high photoactivity under sunlight illumination. Furthermore, the redox potentials for $\mathrm{H}^{+} / \mathrm{H}_{2}$ and $\mathrm{H}_{2} \mathrm{O} / \mathrm{O}_{2}$ must be straddled by valence-band (VB) and conduction-band (CB) edges of the semiconductor material. In addition to the aforementioned requirements, photocatalysts should impede the recombination of the photoexcited electrons and holes, in order to enhance efficiency.

Perovskite structured tantalates with the general formula $A \mathrm{TaO}_{3}$, such as $\mathrm{NaTaO}_{3}$ or $\mathrm{KTaO}_{3}$ [see Figs. 1(a) and 1(b)], have been extensively studied and have shown high chemical stability under (photo)electrochemical conditions, but their light absorption is limited to UV radiation due to their wide band gaps $\left(\mathrm{NaTaO}_{3}=4.1 \mathrm{eV}, \mathrm{KTaO}_{3}=3.6 \mathrm{eV}\right)$ [1-4]. Despite this limitation, tantalates are still considered to be promising materials for water splitting, for instance owing to the development of strategies to reduce their band gaps and therefore increase efficiencies [5,6]. In view of these developments, it is of particular importance to gain a better understanding of the behavior of the excess charge carriers (holes and electrons) in these materials. In photocatalytic applications, the photogenerated electrons and holes need to diffuse efficiently through the material and reach the surface where the reactions are taking place. This process can be disrupted when photogenerated excess charges self-trap and form

\footnotetext{
*julia.wiktor@chalmers.se
}

Published by the American Physical Society under the terms of the Creative Commons Attribution 4.0 International license. Further distribution of this work must maintain attribution to the author(s) and the published article's title, journal citation, and DOI. Funded by Bibsam. localized polarons accompanied by lattice distortion [7,8]. For example, in titanates $\left(\mathrm{SrTiO}_{3}, \mathrm{BaTiO}_{3}, \mathrm{PbTiO}_{3}\right)$, selftrapped (ST) holes/electrons have been observed and studied computationally $[9,10]$. However, only one early computational study [using Hartree-Fock (HF) calculations] has been reported on electron polarons in $\mathrm{KTaO}_{3}[11,12]$ and none for $\mathrm{NaTaO}_{3}$. Accurately predicting properties of self-trapped polarons is challenging with density functional theory (DFT) calculations using semilocal functionals because they suffer from the self-interaction error, which tends to delocalize the electronic states [13-15]. Therefore, modified DFT is required, as applied in hybrid HF/DFT methods, which have been shown to successfully describe charge localization in a wide range of semiconductor materials [16-18].

In this paper, we use hybrid DFT calculations to investigate the formation of self-trapped holes and electrons in the cubic phase of $\mathrm{KTaO}_{3}$ and the orthorhombic phase of $\mathrm{NaTaO}_{3}$. We first determine the fraction $\alpha$ of the Fock exchange that fulfills Koopmans' condition $[19,20]$, hence minimizing the spurious self-interaction. We assess the energetics and the structural

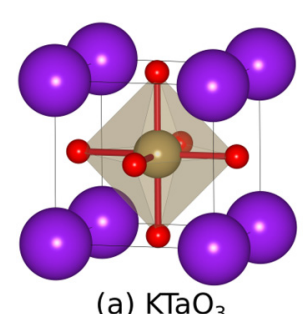

(a) $\mathrm{KTaO}_{3}$

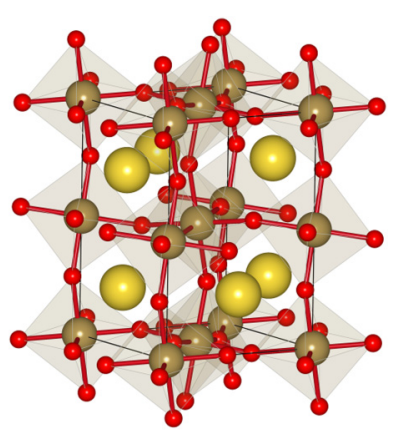

(b) $\mathrm{NaTaO}_{3}$
FIG. 1. Bulk unit cell of (a) cubic $\mathrm{KTaO}_{3}$ with $P m 3 m$ space group and (b) orthorhombic $\mathrm{NaTaO}_{3}$ with $\mathrm{Pbnm}$ space group. The elements $\mathrm{K}, \mathrm{Na}$, Ta, and $\mathrm{O}$ are shown with purple, yellow, brown, and red spheres, respectively. 
distortions associated with the formation of hole and electron polarons, and demonstrate that hole polarons are strongly localized, while electron polarons are distributed within a plane and exhibit a two-dimensional (2D) shape. Finally, by employing the nudged elastic band (NEB) calculation [21], we evaluate the activation energy of hole hopping and the hole mobility in both materials.

\section{COMPUTATIONAL DETAILS}

Here, we use the Perdew-Burke-Ernzerhof $\operatorname{PBEO}(\alpha)$ [22] hybrid functional implemented in the CP2K package [23]. Furthermore, we employ Gaussian-type molecularly optimized (MOLOPT) basis functions [24] with a cutoff energy of 650 Ry for the expansion of the electron density into plane waves. To describe the core-valence interactions, we use the Goedecker-Teter-Hutter pseudopotentials [25]. To improve the performance of Fock exchange calculations, we apply the auxiliary density matrix method (ADMM) [26]. Since hybrid calculations in CP2K use the $\Gamma$ point to sample the Brillouin zone, we perform all calculations using sufficiently large supercells. We employ experimental lattice parameters for cubic $\mathrm{KTaO}_{3}(a=b=c=15.953 \AA)$ and orthorhombic $\mathrm{NaTaO}_{3}$ $(a=10.953, b=11.042$, and $c=15.577 \AA$ ) $[27,28]$.

\section{RESULTS AND DISCUSSION}

\section{A. Fulfillment of Koopmans' conditions}

In this section, we define the fraction $\alpha$ of Fock exchange in $\operatorname{PBEO}(\alpha)$ for $\mathrm{KTaO}_{3}$ and $\mathrm{NaTaO}_{3}$ by verifying the Koopmans' condition. To do so, we calculate the single-particle energy levels induced by the hole polaron and the oxygen vacancy (see Fig. 2). For these calculations we use supercells of 160 atoms for both perovskites. To find the polaronic geometry, we apply the bond distortion method [29] by elongating two $\mathrm{Ta}-\mathrm{O}$ bonds around a specific oxygen site while removing one electron from the system. This distortion leads to the creation of a precursor potential well for localization of the polaron and helps the system to converge easily to the stable polaronic state. After a geometry optimization we find a hole polaron mainly localized at one oxygen atom with a small contribution at four closest oxygen atoms in both materials [see Figs. 3(a) and 3(b)]. This localization is accompanied by an outward displacement of Ta atoms which leads to an elongation of the $\mathrm{Ta}-\mathrm{O}$ bonds. The structures are distorted in such a way that two Ta-O bonds increase by about $0.15 \AA$ for $\mathrm{KTaO}_{3}$ and $0.13 \AA$ for $\mathrm{NaTaO}_{3}$.

The polaronic configurations are then used to calculate the corresponding occupied and unoccupied single-particle levels. The unoccupied state is obtained from the calculation with one electron missing, while the occupied one is found by considering a neutral system in the same geometry. We compute the single-particle levels for two different values of $\alpha, 0.25$ and 0.50 . The crossing between the occupied and unoccupied level corresponds to the value of $\alpha$ for which the Koopmans' condition is satisfied.

It is worth noting that single-particle levels related to a localized state need to be corrected to take into account the electrostatic interactions between both periodic images of the charge and the ionic polarization induced by the lattice distor-
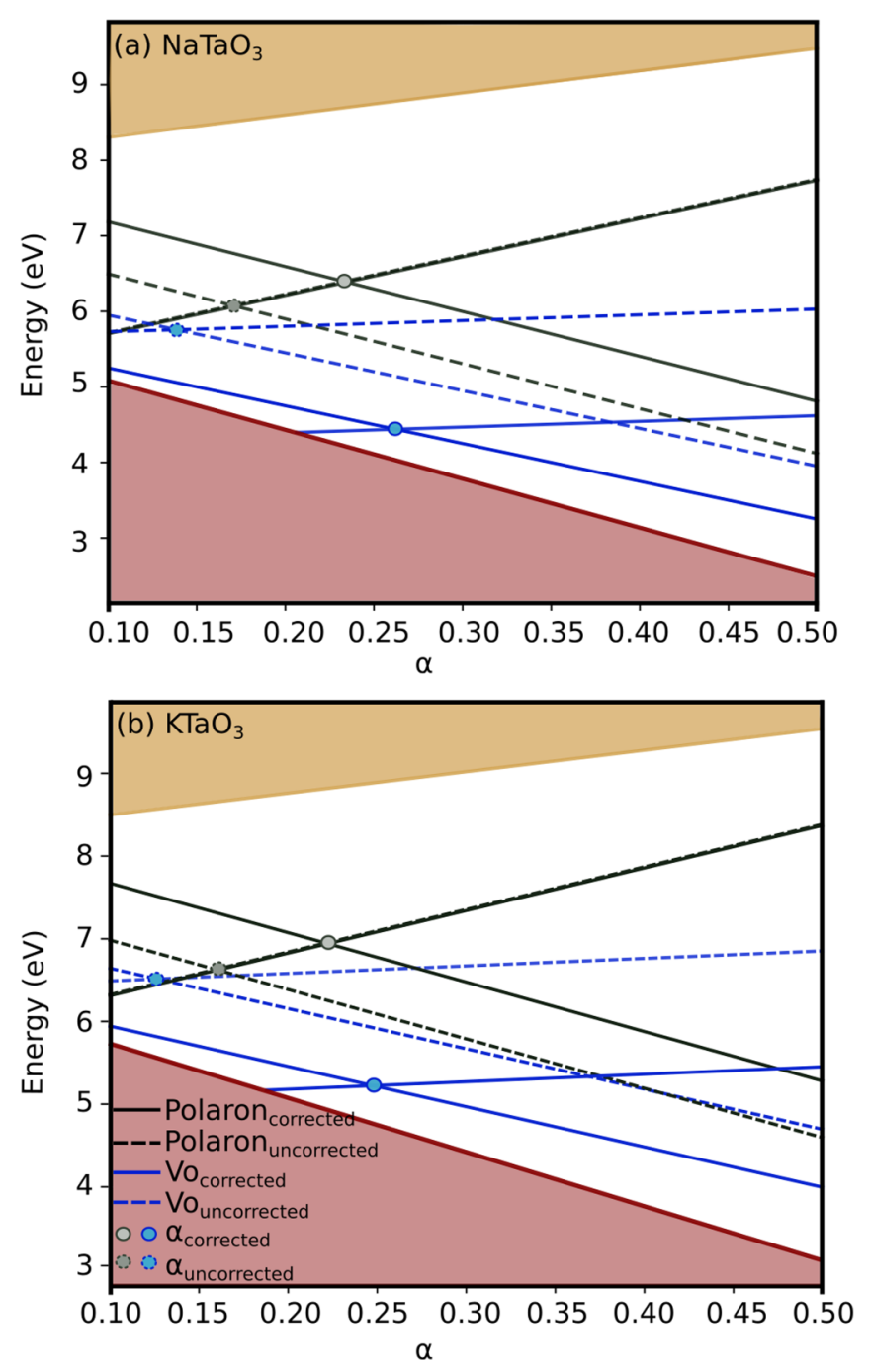

FIG. 2. Band edges and single-particle levels of a hole polaron and oxygen vacancy $\left(V_{\mathrm{O}}\right)$ with respect to the fraction of Fock exchange $\alpha$ in (a) $\mathrm{NaTaO}_{3}$ and (b) $\mathrm{KTaO}_{3}$. The solid lines and circles represent the corrected levels and the corresponding corrected $\alpha$. The dashed lines and circles are uncorrected levels and the uncorrected $\alpha$.

tions. Therefore, we adopt the scheme developed by Falletta et al. [30], to apply finite-size corrections needed to define correctly the single-particle energy levels. We calculate the finite-size corrections using the experimental values of the high-frequency and static dielectric constants $\epsilon_{\infty}$ and $\epsilon_{0}$ of $\mathrm{KTaO}_{3}$ and $\mathrm{NaTaO}_{3}[31,32]$. The corrections are calculated as

$$
\varepsilon_{\mathrm{corr}}^{\mathrm{KS}}=\frac{-2}{q} E_{\mathrm{corr}}\left(q, \epsilon_{0}\right)
$$

for the occupied level, and

$$
\varepsilon_{\mathrm{corr}}^{\mathrm{KS}}=\frac{-2}{q+q_{\mathrm{pol}}^{\prime}} E_{\mathrm{corr}}\left(q+q_{\mathrm{pol}}^{\prime}, \epsilon_{\infty}\right)
$$

for the unoccupied level, where $\varepsilon_{\text {corr }}^{\mathrm{KS}}$ is the correction to the Kohn-Sham (KS) polaron level, and $E_{\text {corr }}\left(q+q_{\text {pol }}^{\prime}, \epsilon_{\infty}\right)$ and $E_{\text {corr }}\left(q, \epsilon_{0}\right)$ are the finite-size electrostatic corrections calculated for the high-frequency and the static dielectric constants, respectively. $q$ of +1 is the charge of the hole polaron, and 
(a)

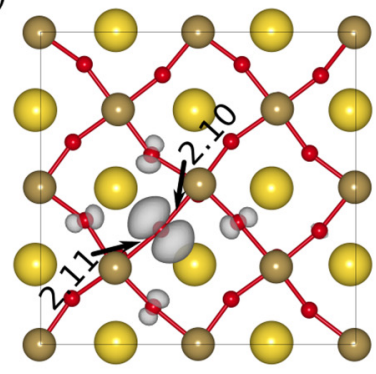

(c)

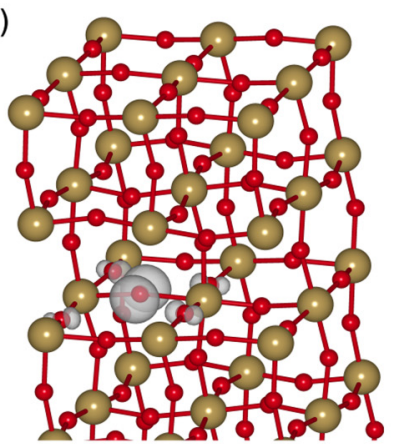

(e)

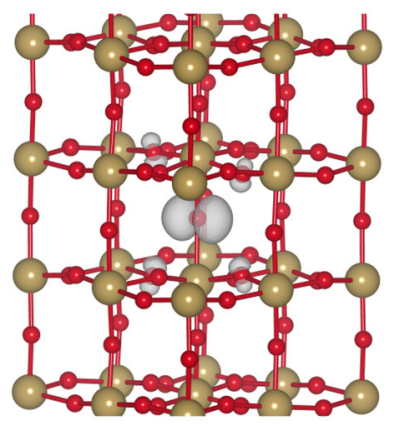

(b)

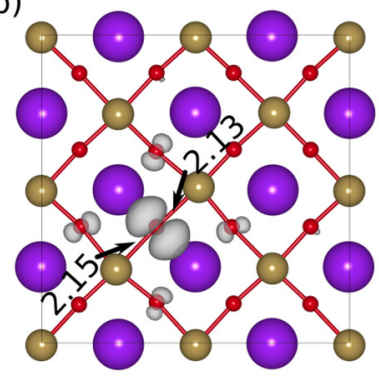

(d)

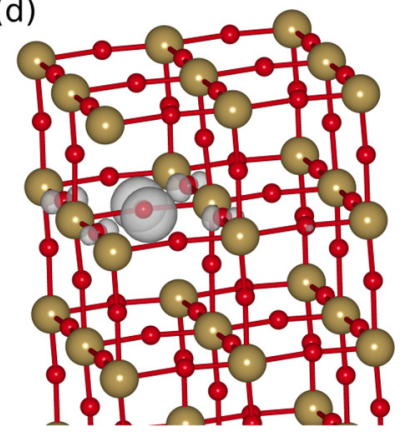

(f)

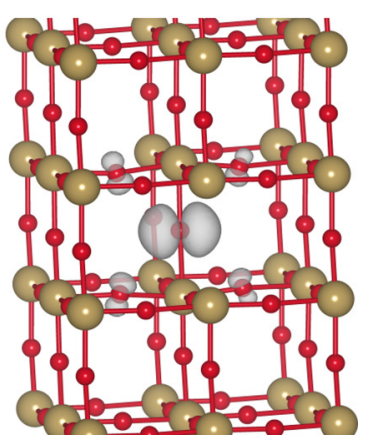

FIG. 3. The spin density isosurface $\left(0.065 \mathrm{e} / \AA^{3}\right)$ representation of (a) top and (c) side views of a self-trapped hole in $\mathrm{NaTaO}_{3}$, (b) top and (d) side views of self-trapped hole in $\mathrm{KTaO}_{3}$, and (e) and (f) side views of the second configuration of a self-trapped hole (parallel to the $z$ direction) in $\mathrm{NaTaO}_{3}$ and $\mathrm{KTaO}_{3}$, respectively. $\mathrm{Na}$ atoms are shown in yellow, $\mathrm{K}$ atoms in purple, Ta atoms in brown, and $\mathrm{O}$ atoms in red. For clarity, we removed the cations ( $\mathrm{Na}$ and $\mathrm{K}$ ) from the side-view figures (c)-(f).

$q_{\mathrm{pol}}^{\prime}$ is the polarization charge caused by the polaronic distortion [30].

Including the corrections shifts the single-particle levels significantly, affecting the value of $\alpha$ for which the Koopmans' condition is fulfilled. For the polaronic distortion, we find $\alpha_{\text {polaron }}=0.23$ for $\mathrm{NaTaO}_{3}$ (significantly larger than the value found before applying electrostatic corrections, $\alpha_{\text {uncorr }}=0.15$ ) [see Fig. 2(a)]. The value of $\alpha_{\text {polaron }}$ is in good agreement with a recently reported $\alpha$ for $\mathrm{NaTaO}_{3}$, calculated as the inverse of the high-frequency dielectric constant $\left(\alpha_{\epsilon_{\infty}}=\right.$ 0.23 ) [33]. For $\mathrm{KTaO}_{3}$, we find $\alpha_{\text {polaron }}=0.22$ [again larger than the uncorrected value $\alpha_{\text {uncorr }}=0.15$; see Fig. 2(b)].

The optimal fraction $\alpha$ should only depend on the material and not the defect type. Therefore, to make the choice of $\alpha$ more robust, we also consider electron localization related to the oxygen vacancy $\left(V_{\mathrm{O}}\right)$. We consider the transition from the formal charge state of this defect $(+2)$, to the one corre-

TABLE I. Dielectric constants $\epsilon_{\infty}$ and $\epsilon_{0}$, and fractions of Fock exchange $\alpha$ that fulfill the Koopmans' condition in $\mathrm{KTaO}_{3}$ and $\mathrm{NaTaO}_{3}$, calculated using uncorrected and corrected positions of the single-particle levels.

\begin{tabular}{|c|c|c|c|c|c|c|}
\hline & \multicolumn{2}{|c|}{ Uncorrected } & \multicolumn{2}{|c|}{ Corrected } & \multicolumn{2}{|c|}{$\epsilon$} \\
\hline & $\alpha_{\text {polaron }}$ & $\alpha_{V_{\mathrm{O}}}$ & $\alpha_{\text {polaron }}$ & $\alpha_{V_{\mathrm{O}}}$ & $\epsilon_{\infty}$ & $\epsilon_{0}$ \\
\hline $\mathrm{KTaO}_{3}$ & 0.15 & 0.12 & 0.22 & 0.24 & 4.35 & 221 \\
\hline $\mathrm{NaTaO}_{3}$ & 0.17 & 0.13 & 0.23 & 0.26 & 4.40 & 200 \\
\hline
\end{tabular}

sponding to the localization of one extra electron on the defect $(+1)$. We perform calculations for the unrelaxed defect (one oxygen atom removed from the pristine cell). As in the case of the hole polaron, we calculate the single-particle energy level for two different values of $\alpha, 0.25$ and 0.50 , in the two considered charge states. Consequently, after considering finite-size corrections, we obtain the values of $\alpha_{V_{0}}=0.24$ and 0.26 for $\mathrm{KTaO}_{3}$ and $\mathrm{NaTaO}_{3}$, respectively.

The calculated values of $\alpha$ which satisfy Koopmans' condition (by hole polaron and oxygen vacancy) are similar. In $\mathrm{NaTaO}_{3}$, the calculated $\alpha$ using $V_{\mathrm{O}}(q=+1$ and +2$)$ is higher by only 0.02 than the one for the hole polaron case, while for $\mathrm{KTaO}_{3}$ the difference is about 0.03 . We note that overall, the fractions of Fock exchange obtained by considering Koopmans' condition in both perovskite oxides are close to the value of 0.25 used in the standard PBE0 functional (see Table I).

\section{B. Electron and hole polarons}

While verifying Koopmans' condition, we focused on the self-trapped hole. We now turn to the case of a self-trapped electron. This is done by adding an electron to a system with an initial distortion where the oxygen bonds around a specific Ta site are elongated. In Fig. 4 we show the spin density of the added electron after a full relaxation, where we see clearly a uniform spread of the spin density over the Ta sites belonging to a single (001) plane for $\mathrm{NaTaO}_{3}$ and to a single (110) plane for $\mathrm{KTaO}_{3}$. We observe that the electron localization has a 2D shape where a fraction of the electron density localizes on different Ta atoms of the same plane in a shape of $d$ orbitals [see Figs. 4(a) and 4(b)]. This is in agreement with the composition of the conduction-band bottom of $\mathrm{NaTaO}_{3}$ and $\mathrm{KTaO}_{3}$ [2,34]. This large electron polaron does not significantly distort the structure, at variance to the case of the hole polaron. The 2D character of the electron polaron is in good agreement with the existence of a $2 \mathrm{D}$ electron gas reported for $\mathrm{KTaO}_{3}, \mathrm{SrTiO}_{3}$ and at interfaces of $\mathrm{SrTiO}_{3} / \mathrm{KTaO}_{3}$ and $\mathrm{SrTiO}_{3} / \mathrm{NaTaO}_{3}$ [35-39]. Additionally, a recent study by Tang et al. (using PBE0 with $\alpha=0.23$ ) reported a similar electron localization behavior for Sr-doped $\mathrm{NaTaO}_{3}$, where substituting $\mathrm{Na}$ by $\mathrm{Sr}$ led to a distribution of the extra electron on all the Ta atoms of the (001) plane close to the $\mathrm{Sr}$ dopant [33].

Having determined the amount of Fock exchange for which the Koopmans' condition is fulfilled, and the polaronic geometries, we can now investigate the energetics of hole and electron polarons in $\mathrm{NaTaO}_{3}$ and $\mathrm{KTaO}_{3}$. To characterize the polaronic states, we calculate their binding energies, which 
(a)

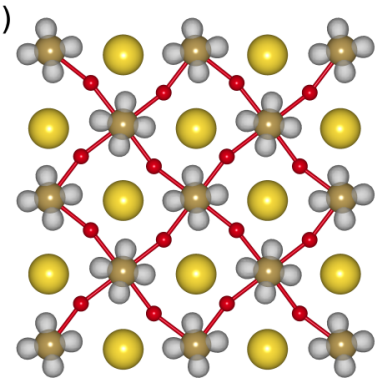

(c)

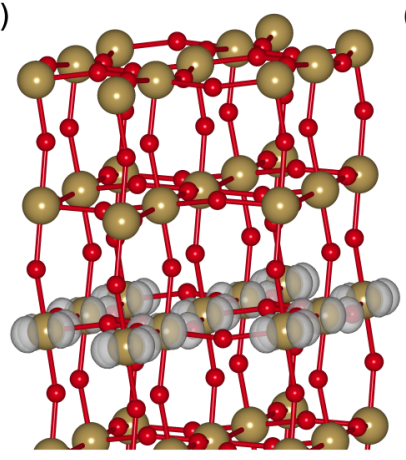

(b)

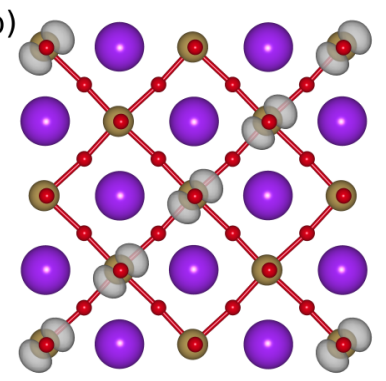

(d)

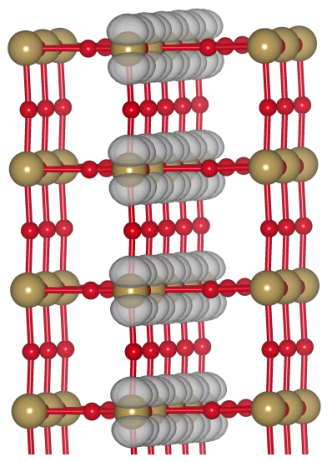

FIG. 4. The spin density isosurface $\left(0.045 e / \AA^{3}\right)$ representation of (a) and (c) top and side views of the 2D shape [plane (001)] of electron localization in $\mathrm{NaTaO}_{3}$, respectively. (b) and (d) Top and side views of the 2D shape [plane (110)] of electron localization in $\mathrm{KTaO}_{3}$, respectively. $\mathrm{Na}$ atoms are shown in yellow, $\mathrm{K}$ atoms in purple, Ta atoms in brown, and $\mathrm{O}$ atoms in red. For clarity, we removed the cations ( $\mathrm{Na}$ and $\mathrm{K}$ ) from the side-view figures (c) and (d).

are defined as follows,

$$
E_{\mathrm{b}}=E_{q}[\text { polaron }]-E_{0}[\text { pristine }]+q \epsilon_{c / v}+E_{\text {corr }},
$$

where $E_{0}$ [pristine] is the energy of the neutral nondistorted structure and $E_{q}$ [polaron] is the energy of the fully relaxed polaronic state, with $q$ denoting the excess of charge ( $q=+1$ for a hole, $q=-1$ for an electron), $\epsilon_{c / v}$ is the position of CBM (for the electron) or the VBM (for the hole), and $E_{\text {corr }}$ is the electrostatic finite-size correction.

In the case of hole polarons, calculated binding energies are negative for both materials. This means that hole localization is preferred to delocalization, and that self-trapped holes are stable in both $\mathrm{KTaO}_{3}$ and $\mathrm{NaTaO}_{3}$. Furthermore, the binding energies in $\mathrm{KTaO}_{3}\left(E_{\mathrm{b}}\right.$ amounting to $\left.-0.97 \mathrm{eV}\right)$ are larger than in $\mathrm{NaTaO}_{3}\left(E_{\mathrm{b}}\right.$ amounting to $\left.-0.58 \mathrm{eV}\right)$. We also note that while in $\mathrm{KTaO}_{3}$ there is only one polaronic state due to the cubic symmetry of the structure [see Fig. 3(f)], in $\mathrm{NaTaO}_{3}$ which is orthorhombic, there are two nonequivalent states. In this case, we find that the state with both elongated $\mathrm{Ta}-\mathrm{O}$ bonds lying in the (001) plane is by $54 \mathrm{meV}$ lower in energy than the one where the elongated bonds are parallel to the $z$ direction [see Fig. 3(e)].

We report binding energies of the hole and electron polarons calculated with different parametrizations of the $\operatorname{PBE0}(\alpha)$ functional in Table II. We note that using the standard PBE0 functional $(\alpha=0.25)$ does not lead to any qualitative change in the hole polaron stability. However, the use of the standard PBE0 method instead of a functional fulfilling Koopmans' condition leads to an overestimation of the

TABLE II. The calculated binding energies for fractions of Fock exchange $\alpha$ that fulfills the Koopmans' condition and the standard value (0.25) for $\mathrm{KTaO}_{3}$ and $\mathrm{NaTaO}_{3}$.

\begin{tabular}{lccccccc}
\hline \hline & \multicolumn{3}{c}{$E_{\mathrm{b}}($ hole) $(\mathrm{eV})$} & & \multicolumn{3}{c}{$E_{\mathrm{b}}$ (electron) $(\mathrm{eV})$} \\
\cline { 2 - 4 } \cline { 6 - 8 } & $\alpha_{\text {polaron }}$ & $\alpha_{V_{\mathrm{O}}}$ & $\alpha_{0.25}$ & & $\alpha_{\text {polaron }}$ & $\alpha_{V_{\mathrm{O}}}$ & $\alpha_{0.25}$ \\
\hline $\mathrm{KTaO}_{3}$ & -0.93 & -1.01 & -1.05 & & -0.44 & -0.43 & -0.43 \\
$\mathrm{NaTaO}_{3}$ & -0.54 & -0.63 & -0.61 & & -0.17 & -0.22 & -0.21 \\
\hline \hline
\end{tabular}

binding energy for $\mathrm{KTaO}_{3}\left(120 \mathrm{meV}\right.$ for $\left.\alpha_{\text {polaron }}\right)$, while the difference is less pronounced in the case of $\mathrm{NaTaO}_{3}(70 \mathrm{meV}$ for $\alpha_{\text {polaron }}$ ).

We now calculate the binding energies of the electron polarons. Despite the fact that electrons only exhibit a weak localization in the considered materials, we find their binding energies to be relatively strong (between about -0.2 and $-0.4 \mathrm{eV}$ ). We also remark that the binding energies of the electron polarons are less affected by the value of the $\alpha$ parameter, compared to the case of the hole polaron (see Table II).

\section{Mobility of polarons}

Strong charge localization, such as the one observed in the case of excess holes in both tantalates, can affect charge mobility and their photocatalytic properties. The most stable surfaces of $\mathrm{NaTaO}_{3}$ and $\mathrm{KTaO}_{3}$ are (001)-oriented [40,41], therefore, we assess the activation energy for hole hopping along the (001) direction between the most stable configurations through nudged elastic band (NEB) calculations [21]. We note that in principle the energy barrier calculated from NEB could be affected by finite-size corrections. However, in the present case the charge remains localized at all steps along the path, meaning that the corrections for all configurations should be close and largely cancel each other out. Moreover, with dielectric constants $\epsilon_{0}$ of both materials being large (see Table I) the finite-size corrections are very small (the order of $0.01 \mathrm{eV}$ ). Therefore, even if they do not cancel out exactly, they do not significantly affect our NEB calculations. In $\mathrm{KTaO}_{3}$, hopping along the direction (001) can take place along two different paths. One corresponds to the polaron hopping directly between the neighboring $\mathrm{TaO}_{2}$ planes (A to $\mathrm{B}$ in Fig. 5), with the energy barrier of $340 \mathrm{meV}$. The second one involves hopping between $\mathrm{TaO}_{2}$ and $\mathrm{NaO}$ layers ( $\mathrm{C}$ to $\mathrm{D}$ in Fig. 5), with a similar energy barrier of $348 \mathrm{meV}$. In the case of the orthorhombic $\mathrm{NaTaO}_{3}$, hopping between the most stable oxygen sites along the (001) direction involves two different pathways to migrate from one $\mathrm{TaO}_{2}$ layer to another, a longer one ( $\mathrm{C}$ to A with the energy barrier of $248 \mathrm{meV}$ ) of $4.28 \AA$ and a shorter one (A to B with the energy barrier of $219 \mathrm{meV}$ ) of $3.49 \AA$. In addition, we observe that the charge is transferred between the two oxygens through a transition state where the hole is localized at the final polaronic site, which is consistent with the nonadiabatic hopping.

Next, according to the Emin-Holstein formalism [42], in the transition region between the two domains (nonadiabatic and adiabatic), the nonadiabatic hopping probability is comparable to its adiabatic counterpart. Therefore, we estimate the hole mobility $(\mu)[43-45]$ in both materials as

$$
\mu=\left[e a^{2} \omega_{0} / k_{B} T\right] \exp \left(-E_{\mathrm{a}} / k_{B} T\right),
$$



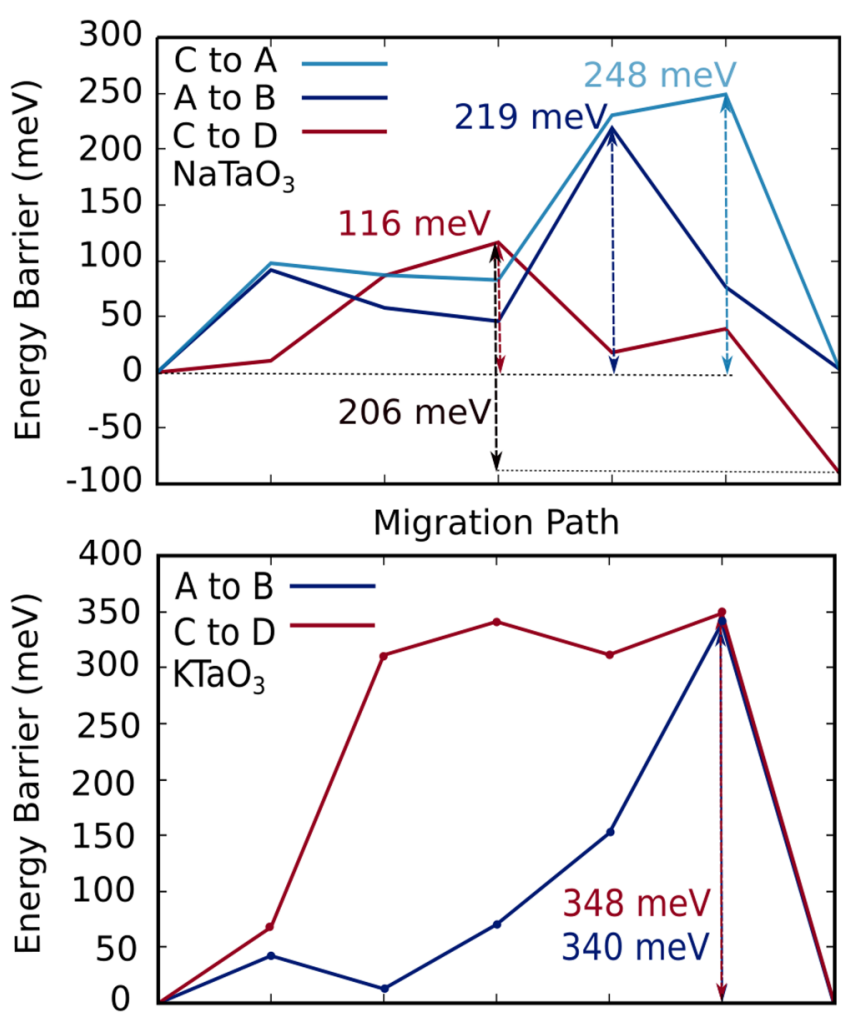

Migration Path
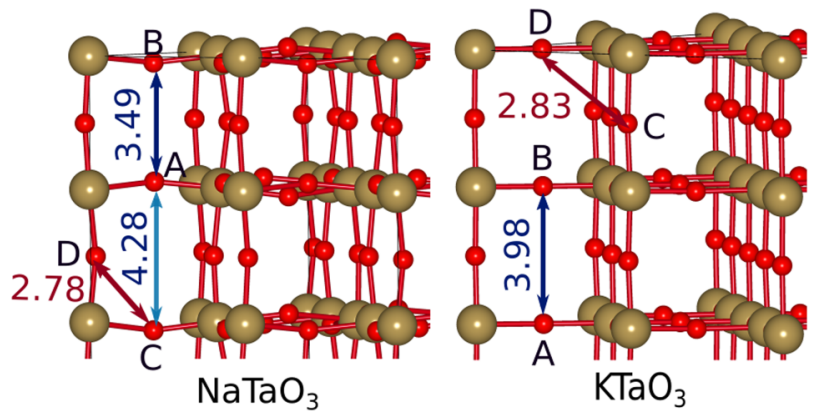

FIG. 5. The migration energy barriers of a hole polaron for the considered migration pathways in $\mathrm{NaTaO}_{3}$ and $\mathrm{KTaO}_{3}$.

where $a$ is the hopping distance between the two oxygen sites (A to B), $k_{B}$ is the Boltzmann's constant, $T$ is the temperature, $\omega_{0}$ is the longitudinal phonon frequency, and $E_{\mathrm{a}}$ is the hopping activation energy. Through this formula, we find the hole mobility at room temperature $(300 \mathrm{~K})$ in $\mathrm{KTaO}_{3}$ to amount to $2.94 \times 10^{-6} \mathrm{~cm}^{2} / \mathrm{Vs}$, for $E_{\mathrm{a}}=340 \mathrm{meV}$, $a=3.98 \AA$, and $\omega_{0}=826 \mathrm{~cm}^{-1}$ [46]. For $\mathrm{NaTaO}_{3}$, the hole mobility is $2.52 \times 10^{-4} \mathrm{~cm}^{2} / \mathrm{Vs}$ for the longest pathway ( $a=$ $4.28 \AA, E_{\mathrm{a}}=248 \mathrm{meV}$ ), and $1.23 \times 10^{-4} \mathrm{~cm}^{2} / \mathrm{Vs}$ for the shortest pathway $\left(a=3.49 \AA, E_{\mathrm{a}}=219 \mathrm{meV}\right)$ at room temperature and $\omega_{0}=855 \mathrm{~cm}^{-1}$ [31]. Our estimations of the hole mobilities show that the holes are more mobile in $\mathrm{NaTaO}_{3}$ than $\mathrm{KTaO}_{3}$, which is consistent with the more localized hole state in $\mathrm{KTaO}_{3}$ and the lower photocatalytic activity compared to $\mathrm{NaTaO}_{3}$ [47]. To the best of our knowledge, the experimental values of carriers mobility are unfortunately lacking for $\mathrm{NaTaO}_{3}$, and $\mathrm{KTaO}_{3}$. As for the electron polaron, its two-dimensional localization indicates that the hopping mechanism cannot be applied. However, we expect that while the mobility of electrons in the direction normal to the polaron density will be limited, they can easily migrate to the surface in the parallel direction, which could be beneficial for photocatalytic reactions [48]. This predicted high mobility of electrons in $\mathrm{NaTaO}_{3}$ and $\mathrm{KTaO}_{3}$ is in contrast to what was modeled in a non-perovskite tantalum-based oxide and nitrides, where the electron polaron mainly localizes at one Ta site, leading to mobilities of $9.41 \times 10^{-5} \mathrm{~cm}^{2} / \mathrm{Vs}$ (for $\beta$ $\mathrm{TaON}$ ) and $9.96 \times 10^{-4} \mathrm{~cm}^{2} / \mathrm{Vs}\left(\right.$ for $\mathrm{Ta}_{3} \mathrm{~N}_{5}$ ) $[44,49]$.

\section{CONCLUSIONS}

In the present study, we have investigated the properties of the excess holes and electrons in $\mathrm{NaTaO}_{3}$ and $\mathrm{KTaO}_{3}$. First, we showed the importance of fulfilling the Koopmans' conditions and finite-size corrections in a hybrid functional $\operatorname{PBE0}(\alpha)$-based study. We adopted two types of defects, a hole polaron and an oxygen vacancy to determine the fraction $\alpha$ of the Fock exchange. Next, we showed that small hole polarons are formed in both $\mathrm{NaTaO}_{3}$ and $\mathrm{KTaO}_{3}$. The self-trapped holes are localized mainly on one oxygen atom, with a small fraction of density spread over four neighboring oxygen atoms. This localization is accompanied by structural distortions where $\mathrm{Ta}-\mathrm{O}$ bonds are elongated by about $0.13 \AA$ for $\mathrm{NaTaO}_{3}$ and $0.15 \AA$ for $\mathrm{KTaO}_{3}$. We estimated that a hole polaron in $\mathrm{KTaO}_{3}$ has a mobility of $2.94 \times 10^{-6} \mathrm{~cm}^{2} / \mathrm{Vs}$ at room temperature which is two orders of magnitude lower than that of $\mathrm{NaTaO}_{3}$ because of its stronger binding energy. In contrast to the small hole polarons, electron polarons were found to be larger and distributed on different Ta atoms belonging to the same plane, in a 2D-electron-gas-like shape. Overall, our results have implications for application of tantalate perovskites in photocatalysis. On the one hand, the low mobility of the hole polaron compared to the electron could retard the electron-hole recombination, but on the other hand, it limits the diffusion of the holes to the surface and therefore reduces its activity. Finally, our study lays the groundwork for further experimental or theoretical investigations to deepen our understanding of polaron transport in tantalate perovskites.

\section{ACKNOWLEDGMENTS}

The authors acknowledge funding from the "Area of Advance - Materials Science" at Chalmers University of Technology, and the Swedish Research Council (2019-03993). The computations were performed on resources provided by the Swedish National Infrastructure for Computing (SNIC) at NSC, C3SE, and PDC. This work was supported by Chalmers Gender Initiative for Excellence (Genie).
[1] E. Grabowska, Appl. Catal., B 186, 97 (2016).

[2] B. Modakand S. K. Ghosh, J. Phys. Chem. C 120, 6920 (2016).
[3] H. Kato and A. Kudo, J. Phys. Chem. B 105, 4285 (2001).

[4] H. Kato, K. Asakura, and A. Kudo, J. Am. Chem. Soc. 125, 3082 (2003). 
[5] B. Modak and S. K. Ghosh, Sol. Energy Mater. Sol. Cells 159, 590 (2017).

[6] B. Wang, P. D. Kanhere, Z. Chen, J. Nisar, B. Pathak, and R. Ahuja, J. Phys. Chem. C 117, 22518 (2013).

[7] H. Frhlich, Adv. Phys. 3, 325 (1954).

[8] D. Emin, Phys. Rev. B 48, 13691 (1993).

[9] P. Erhart, A. Klein, D. Åberg, and B. Sadigh, Phys. Rev. B 90, 035204 (2014).

[10] X. Hao, Z. Wang, M. Schmid, U. Diebold, and C. Franchini, Phys. Rev. B 91, 085204 (2015).

[11] E. A. Kotomin, R. I. Eglitis, and G. Borstel, J. Phys.: Condens. Matter 12, L557 (2000).

[12] O. Bidault, M. Maglione, M. Actis, M. Kchikech, and B. Salce, Phys. Rev. B 52, 4191 (1995).

[13] W. Chen and A. Pasquarello, Phys. Rev. B 88, 115104 (2013).

[14] J. Lægsgaard and K. Stokbro, Phys. Rev. Lett. 86, 2834 (2001).

[15] S. Lany and A. Zunger, Phys. Rev. B 80, 085202 (2009).

[16] M. Choi, F. Oba, and I. Tanaka, Phys. Rev. B 83, 214107 (2011).

[17] E. Bousquet, H. Hamdi, P. Aguado-Puente, E. K. H. Salje, E. Artacho, and P. Ghosez, Phys. Rev. Research 2, 012052(R) (2020).

[18] A. Janotti, J. B. Varley, M. Choi, and C. G. Van de Walle, Phys. Rev. B 90, 085202 (2014).

[19] T. Bischoff, J. Wiktor, W. Chen, and A. Pasquarello, Phys. Rev. Materials 3, 123802 (2019).

[20] I. Dabo, A. Ferretti, N. Poilvert, Y. Li, N. Marzari, and M. Cococcioni, Phys. Rev. B 82, 115121 (2010).

[21] N. A. Zarkevich and D. D. Johnson, J. Chem. Phys. 142, 024106 (2015).

[22] J. P. Perdew, M. Ernzerhof, and K. Burke, J. Chem. Phys. 105, 9982 (1996).

[23] J. VandeVondele, M. Krack, F. Mohamed, M. Parrinello, T. Chassaing, and J. Hutter, Comput. Phys. Commun. 167, 103 (2005).

[24] J. VandeVondele and J. Hutter, J. Chem. Phys. 127, 114105 (2007).

[25] S. Goedecker, M. Teter, and J. Hutter, Phys. Rev. B 54, 1703 (1996).

[26] M. Guidon, J. Hutter, and J. VandeVondele, J. Chem. Theory Comput. 6, 2348 (2010).

[27] B. J. Kennedy, A. K. Prodjosantoso, and C. J. Howard, J. Phys.: Condens. Matter 11, 6319 (1999).
[28] J. Sigman, D. P. Norton, H. M. Christen, P. H. Fleming, and L. A. Boatner, Phys. Rev. Lett. 88, 097601 (2002).

[29] T. D. Pham and N. A. Deskins, J. Chem. Theory Comput. 16, 5264 (2020).

[30] S. Falletta, J. Wiktor, and A. Pasquarello, Phys. Rev. B 102, 041115(R) (2020).

[31] S. Kamba, V. Goian, V. Bovtun, D. Nuzhnyy, M. Kempa, M. Spreitzer, J. Knig, and D. Suvorov, Ferroelectrics 426, 206 (2012).

[32] R. C. Miller and W. G. Spitzer, Phys. Rev. 129, 94 (1963).

[33] Z.-K. Tang, C. Di Valentin, X. Zhao, L.-M. Liu, and A. Selloni, ACS Catal. 9, 10528 (2019).

[34] H. Ouhbi and U. Aschauer, Surf. Sci. 677, 258 (2018).

[35] S. Nazir and U. Schwingenschlgl, Appl. Phys. Lett. 99, 073102 (2011).

[36] I. V. Maznichenko, S. Ostanin, A. Ernst, J. Henk, and I. Mertig, Phys. Status Solidi B 257, 1900540 (2020).

[37] H. Zhang, X. Yan, X. Zhang, S. Wang, C. Xiong, H. Zhang, S. Qi, J. Zhang, F. Han, N. Wu, B. Liu, Y. Chen, B. Shen, and J. Sun, ACS Nano 13, 609 (2019).

[38] F. Y. Bruno, S. McKeown Walker, S. Ricc, A. de la Torre, Z. Wang, A. Tamai, T. K. Kim, M. Hoesch, M. S. Bahramy, and F. Baumberger, Adv. Electron. Mater. 5, 1800860 (2019).

[39] G. R. Portugal and J. T. Arantes, Nano Express 2, 010016 (2021).

[40] M. Setvin, M. Reticcioli, F. Poelzleitner, J. Hulva, M. Schmid, L. A. Boatner, C. Franchini, and U. Diebold, Science 359, 572 (2018).

[41] H. Ouhbi and U. Aschauer, J. Mater. Chem. A 7, 16770 (2019).

[42] D. Emin and T. Holstein, Ann. Phys. 53, 439 (1969).

[43] I. G. Austin and N. F. Mott, Adv. Phys. 50, 757 (2001).

[44] M. Dey, A. Singh, and A. K. Singh, J. Phys. Chem. C 125, 11548 (2021).

[45] J. Lago, P. D. Battle, M. J. Rosseinsky, A. I. Coldea, and J. Singleton, J. Phys.: Condens. Matter 15, 6817 (2003).

[46] H. Vogt, Phys. Rev. B 38, 5699 (1988).

[47] H. Onishi, ChemSusChem 12, 1825 (2019).

[48] F.-N. Wang, J.-C. Li, Y. Li, X.-M. Zhang, X.-J. Wang, Y.-F. Chen, J. Liu, C.-L. Wang, M.-L. Zhao, and L.-M. Mei, Chin. Phys. B 28, 047101 (2019).

[49] J. M. Morbec and G. Galli, Phys. Rev. B 93, 035201 (2016). 